\title{
Application of a Telescoping Microextraction Needle Trap Sampling Device on a Drone to Extract Airborne Organic Vapors
}

\author{
Wen-Hsi Cheng $^{1 *}$, Tso-Sheng Hsieh ${ }^{2}$, Chun-Mou Chu ${ }^{3}$, Chia-Chin Chiang ${ }^{2}$, Chung-Shin Yuan ${ }^{3}$ \\ ${ }^{1}$ Department of Occupational Safety and Hygiene, Fooyin University, Kaohsiung 83102, Taiwan \\ ${ }^{2}$ Department of Mechanical Engineering, National Kaohsiung University of Science and Technology, Kaohsiung 80778, \\ Taiwan \\ ${ }^{3}$ Institute of Environmental Engineering, National Sun Yat-Sen University, Kaohsiung 80424, Taiwan
}

\begin{abstract}
A quadrotor drone was equipped with an active solid phase microextraction (SPME) sampling device, known as a needle trap sampler (NTS), for extracting airborne volatile organic compounds (VOCs). The NTS, which has a 22-gauge stainless steel needle that is manually packed with 60-80-mesh adsorbent divinylbenzene (DVB) particles, was attached to a telescoping shaft to extend the sampling range beyond the strong downward stream that flows across the propellers of a hovering drone. The end of the NTS inlet was positioned below the head of the drone, which was the optimal location determined by using the flow simulation software SolidWorks. Our results confirm the feasibility of collecting toluene, ethylbenzene, and $p$-xylene matrix vapor with a telescoping SPME sampling device on a quadrotor drone using a pilot VOC exhaustion system. Therefore, we recommend this mobile system for sampling polluted air emitted by industrial factories.
\end{abstract}

Keywords: Solid phase microextraction; Needle trap sampler; Drone; Volatile organic compounds; Air pollution.

\section{INTRODUCTION}

Industrial air pollution greatly affects human lives and the environment. If pollutants are not detected in a timely manner, then follow-up treatments will not be effectively implemented. In Taiwan, which is a small and densely populated island, residential areas are close to industrial sources of air pollution, negatively affecting the quality of life. Technology for monitoring the atmospheric environment using remote sensors and drones has recently been developed (Lähde et al., 2014; Syu et al., 2016; Xu et al., 2017; Cheng, 2018), and unmanned drones have been used in places that are difficult to access, such as volcanoes (McGonigle et al., 2008), high mountains (Chang et al., 2016), sea coasts (Chang et al., 2018), and areas where open burning is occurring (Aurell et al., 2017). Using a high-mobility drone to sample immediately and continuously odorous and hazardous air emissions and then returning it to the ground to enable the collected samples to be examined in a laboratory can significantly reduce the emission of pollution.

\footnotetext{
* Corresponding author.

Tel.: +886-7-781-1151 ext. 5113; Fax: +886-7-782-6735

E-mail address: PL031@fy.edu.tw
}

Unmanned drones that carry atmospheric monitoring sensors with a data logger typically record sampling times, altitudes, GPS coordinates, relative humidity, and temperature, as well as $\mathrm{PM}_{1.0}, \mathrm{PM}_{2.5}, \mathrm{PM}_{10}$, carbon monoxide, nitric oxide, sulfur dioxide, and total volatile organic compound (TVOC) concentrations (Peng et al., 2015; Villa et al., 2016; Schuyler and Guzman, 2017). Data are recorded and transmitted wirelessly at a rate up to 3-6 data per minute, and organic pollutants are detected as the concentrations of TVOC. Users are therefore unable to identify contaminants and locate the source of the pollution.

Many researchers have used drones, carrying either stainless steel canisters with various volumes (400-2,000 mL) and a remote-controlled on-off solenoid valve system (Chang et al., 2016; Chang et al., 2018; Vo et al., 2018), or a charcoal tube that is connected to a sampling pump (Aurell et al., 2017), to collect aerial samples. Collected gas samples can be qualitatively and quantitatively analyzed in a laboratory using a gas chromatograph (GC) that is connected to a mass spectrometric (MS) detector. However, the required Li battery, canister, sensors and other sampling and electrical accessories must be loaded on a multirotor drone, such as a 1,045-mm diagonal wheelbase octorotor multicopter (Spreading Wings S1000; DJI), which has a take-off mass of $11 \mathrm{~kg}$ (Chang et al., 2016, 2018).

Solid-phase microextraction (SPME) sampling technology has been used to collect gaseous VOCs, which are 
thermally desorbed when the sampling fiber is injected into the inlet of a gas chromatograph (GC). Cheng et al. (2011) sampled gaseous aromatic compounds using divinylbenzene (DVB) adsorbent that was packed in specially made needle-type SPME devices, called needle trap samplers (NTSs; Fig. 1). They successfully sampled various emissions from indoor sources (Cheng et al., 2013; Cheng and Lai, 2014; Cheng et al., 2014, 2015, 2016) and organic vapor emissions in workplaces (Cheng et al., 2017, 2019). In this work, an NTS and an air-extracting pump were installed on a light and mini-sized quadrotor drone (Mavic Pro; DJI) (Fig. 2) to measure ambient organic emissions from stationary air exhaust sources. This quadrotor multicopter is very small, and it can travel faster and more safely than an octorotor drone between exhaust stacks in industrial factories. More importantly, the air turbulence that is generated by drone propellers has seldom been studied except by Zhou et al. (2018), who suggested that the sampling position should be less influenced by the air flow generated by the rotation of the rotors. In this investigation, the pattern of air flow when a quadrotor drone travels through sprayed dry ice was observed, and then SolidWorks (2018) was used to simulate the flow streams to optimize precisely the sampling locations around the drone. Based on the results of the model simulation, a specially designed telescoping NTS sampling device was used to collect non-disturbed samples exhausted from a stack.

\section{METHOD}

\section{Specifications of Unmanned Drone}

The Mavic Pro quadrotor drone, manufactured by DJI (Fig. 2), was used as the atmospheric sampling vehicle. It is so small that it can be flown through factory chimneys. It has a 335-mm diagonal wheelbase (excluding propellers), a maximum horizontal voyage speed of $65 \mathrm{~km} \mathrm{~h}^{-1}$, and a 21-min operating time with a minimum residual battery electric power of $15 \%$. A telescoping sampling device, including an NTS, was connected to a Teflon sampling tube and a 6-V-DC air-extracting pump. All components are attached on the plastic frames, which are installed on the ventral part of the drone (Fig. 3). The air-extracting pump is operated using an on-off switch via the remote drone controller. This target drone is recommended by its manufacturer for performing flights except during rain and when the wind speed exceeds $10 \mathrm{~m} \mathrm{~s}^{-1}$.

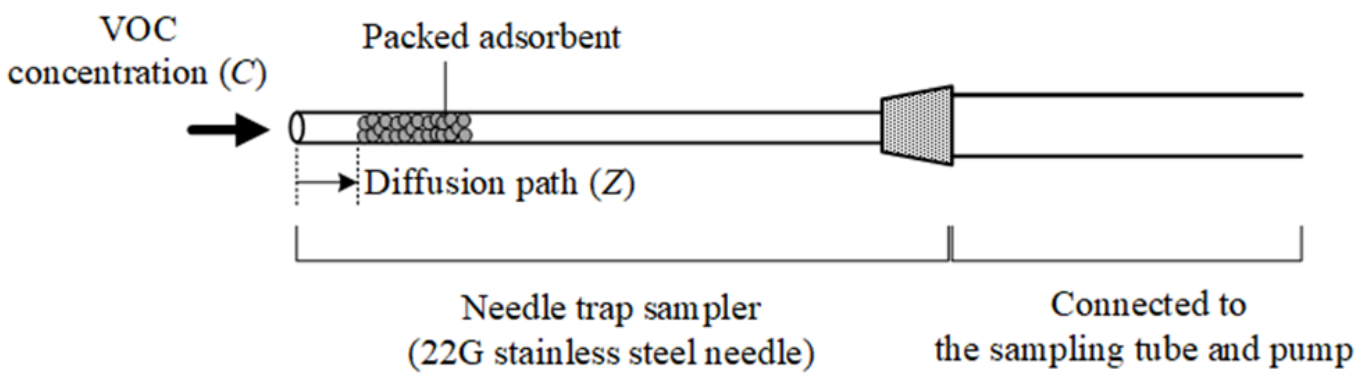

Fig. 1. Schematic needle trap sampler.

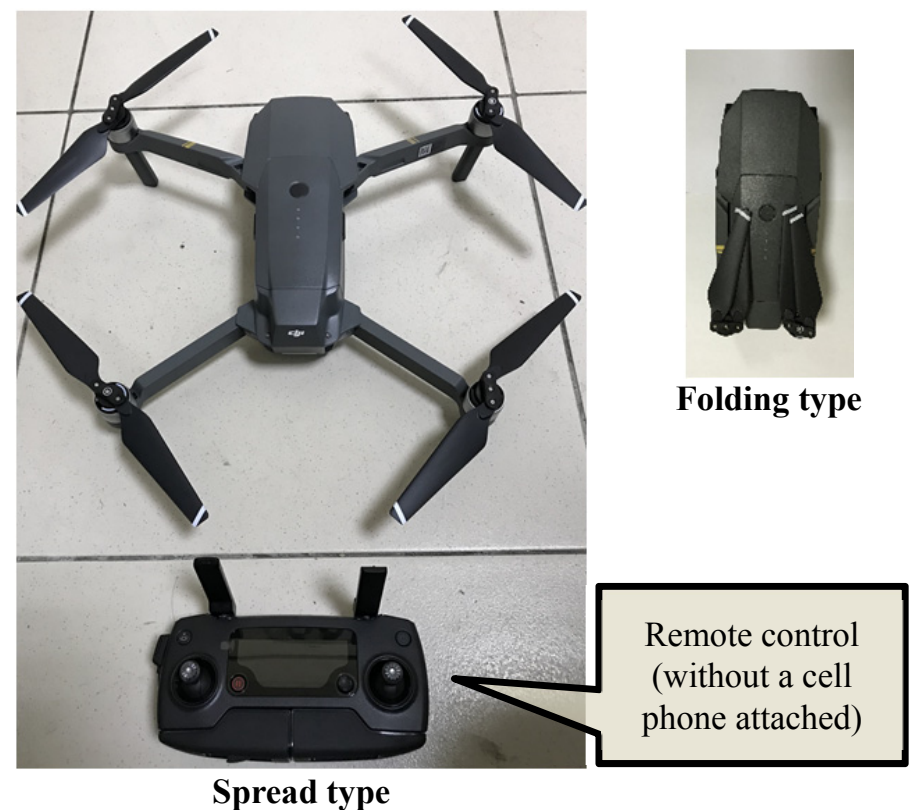

Fig. 2. Outlines and dimensions of the quadrotor drone used in this work: spread type: 335-mm-diagonal wheelbase, excluding propellers; folding type: $83 \mathrm{~mm}(\mathrm{~W}) \times 198 \mathrm{~mm}(\mathrm{~L}) \times 83 \mathrm{~mm}(\mathrm{H})$. 


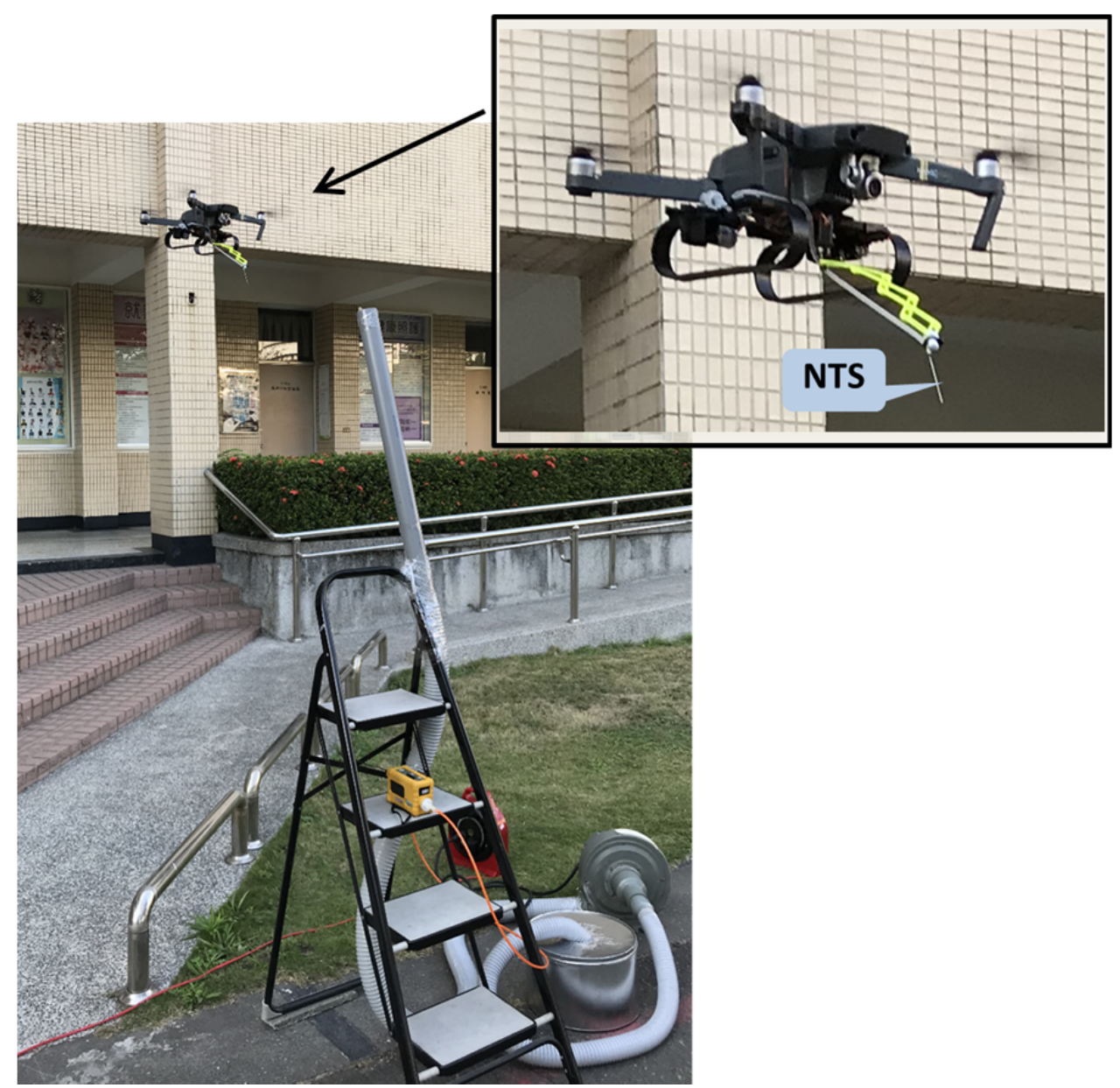

Fig. 3. Pilot experiment set-up for the VOC exhaustion system.

\section{Simulation of Air Stream Generated by Propellers of Drone}

The simulated air was assumed to be compressible, in a steady state, and a homogeneous laminar Newtonian fluid. Flows in the model with moving walls (without changes to the model geometry) were computed under specified corresponding boundary conditions. Flows in models with rotating parts were computed in the coordinate systems, so the models' stationary parts were axisymmetric with respect to the axis of rotation.

The conservation of mass, angular momentum, and energy in the Cartesian coordinate system, rotating with angular velocity $\Omega$ about an axis that passes through the coordinate system's origin can be expressed as follows (SolidWorks, 2018).

Mass Equation:

$\frac{\partial \rho}{\partial t}+\frac{\partial}{\partial x_{i}}\left(\rho u_{i}\right)=0$

Angular Momentum Equation:

$\frac{\partial u_{i}}{\partial t}+\frac{\partial}{\partial x_{j}}\left(\rho u_{i} u_{j}\right)=-\frac{\partial p}{\partial x_{i}}+\frac{\partial\left(\tau_{i j}+\tau_{i j}^{R}\right)}{\partial x_{j}}+S_{i} \quad i=1,2,3$
Energy Equation:

$$
\begin{aligned}
\frac{\partial \rho H}{\partial t}+\frac{\partial \rho u_{i} H}{\partial x_{i}} & =\frac{\partial}{\partial x_{i}}\left(u_{j}\left(\tau_{i j}+\tau_{i j}^{R}\right)+q_{i}\right)+\frac{\partial p}{\partial t}-\tau_{i j}^{R} \frac{\partial u_{i}}{\partial x_{j}} \\
& +\rho \varepsilon+S_{i} u_{i} \frac{\partial \rho H}{\partial t}+\frac{\partial \rho u_{i} H}{\partial x_{i}} \\
& =\frac{\partial}{\partial x_{i}}\left(u_{j}\left(\tau_{i j}+\tau_{i j}^{R}\right)+q_{i}\right)+\frac{\partial p}{\partial t}-\tau_{i j}^{R} \frac{\partial u_{i}}{\partial x_{j}} \\
& +\rho \varepsilon+S_{i} u_{i}
\end{aligned}
$$

where $u$ is the fluid velocity; $\rho$ is the fluid density; $S_{i}$ is a mass-distributed external force per unit mass due to a buoyancy; $\mathrm{h}$ is the thermal enthalpy, and $\mathrm{H}=\mathrm{h}+u^{2} / 2 . \tau_{i k}$ is the viscous shear stress tensor. The subscripts are used to denote summation over the three coordinate directions.

The velocity and turbulence intensity of the flow streams around the drone were analyzed using the flow simulation software SolidWorks (2018). Original size (1:1) modeling was performed, and the boundary condition corresponded to a Quad X configuration of the rotating propellers of the drone. The adjacent propellers rotated oppositely, as shown in Fig. 4. Four rotating regions were identified in the model, and the angular velocity of the 


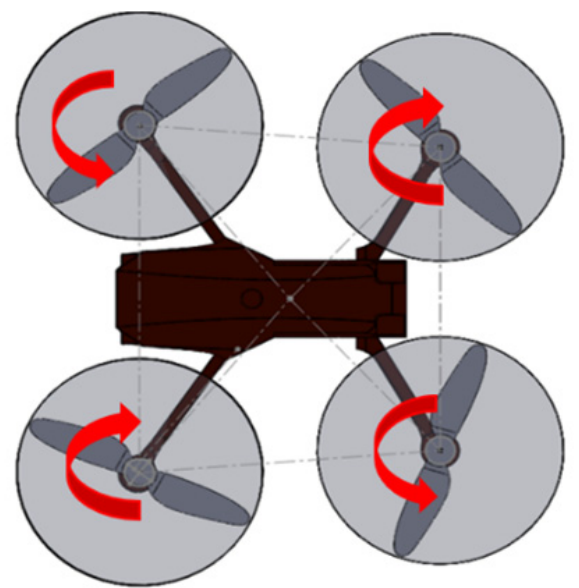

Fig. 4. Schematic diagram of original-size model.

propeller was set to $5,700 \mathrm{rpm}$, as measured for the drone. The initial unit mesh used in the simulation model was set by default to 3 , and the total number of cells was 9,963 .

\section{Gaseous Sampling System}

An NTS extracts organic compounds from the air through a needle by diffusion or by drawing via a pump. Therefore, a linear gaseous concentration profile $(C(Z)$ in Fig. 1) is obtained along the diffusion path $(Z)$, and the extraction is characterized by the area $(A)$ of the opening and the diffusion path length. The total amount $(n)$ of analyte that is extracted in a time interval $(t)$ is estimated using the following formula (Lord et al., 2010):

$$
n=D_{m} \frac{A}{Z} \int C(t) d t
$$

where $D_{m}$ is the diffusion coefficient of a compound that is sampled by the sorbent in the needle. The amount $(n)$ of the extracted analyte is assumed to be proportional to the total sample concentration over a time interval $(C(t))$ given a constant $D_{m}$, a uniform needle opening $(A)$, and a fixed diffusion path distance $(Z)$. The detailed calculation procedures for concentrations of VOCs and the calibration method are presented in the "Supplement" section.

The NTS comprised 22-G stainless steel needles (OD = $0.71 \mathrm{~mm}$ ), which were purchased from a local company (Herling Co. Ltd., Pingtung, Taiwan), and 60-80-mesh DVB particles (Supelco, Bellefonte, PA, USA). The DVBNTS was prepared and tested as described elsewhere (Cheng et al., 2013; Cheng and Lai, 2014; Cheng et al., 2014, 2015, 2016, 2017, 2019).

\section{Analysis and Instruments}

Gaseous samples were analyzed using a GC (6890N; Agilent, Wilmington, DE, USA), which was equipped with a flame ionization detector (FID). The capillary column was an HP-1 PDMS $(30 \mathrm{~m} \times 320 \mu \mathrm{m} \times 0.25 \mu \mathrm{m}$, part no. HP19091Z-413; Agilent Technologies, Inc., Wilmington, DE, USA). All gases (Jing-De Gas Co., Ltd., Kaohsiung, Taiwan) that were used in the chromatographic analysis were of ultra-high purity. The VOC analysis of both the calibrations and air samplings of the NTS and sampling bags were also performed using the same GC-FID. The NTS calibration procedures for VOCs are displayed in "Supplement," and the calibration procedures for sampling bags also followed the same method. After VOC sampling, NTS were injected into the injection ports of GC used for VOC desorption and further analysis. The desorption time at the injection port of GC was $30 \mathrm{~s}$. The temperature of the $\mathrm{GC}$ increased from $50^{\circ} \mathrm{C}$ at increments of $15^{\circ} \mathrm{C} \mathrm{min}^{-1}$. The final temperature was held at $180^{\circ} \mathrm{C}$ for $2 \mathrm{~min}$. The FID detector was heated to $300^{\circ} \mathrm{C}$. The carrying gas flow rate was $1.2 \mathrm{~mL} \mathrm{~min}^{-1}$ for nitrogen, and the split off operation mode was selected. Notably, after the operation and subsequent analysis, no carryovers were available for sample extraction by NTS. Analysis procedures for the air samples, which were taken using a syringe from Teflon sampling bags, were all the same as NTS analysis procedures by GC-FID.

\section{Pilot test for Sampling and Analysis}

The pilot sampling experiment was carried out using an industrial solvent that is commonly used in the local electronic processing industry. The liquid compounds of toluene, ethylbenzene, and $p$-xylene were mixed together around $1,500 \mathrm{~mL}$ with the volume ratio as 1:1:1. A $0.75-\mathrm{Hp}$ exhaust fan of 3,500 rpm (Teco Electric \& Machinery, Taiwan) connected to a hose pipe was used to ventilate the air that was emitted from the industrial solvent (Fig. 3). For $15 \mathrm{~min}$, the drone, carrying the telescoping SPME sampling device, was used to collect samples of air that had been exhausted from the PVC chimney. When the NTS was used to collect the air samples, a VOC monitor (MultiRAE; RAE Systems Inc., CA, USA) was simultaneously used to measure the emission concentrations of VOCs from the chimney. Additional air samples were obtained using Teflon sampling bags and an air collection pump for a comparison of the sampling performance of the NTS on the unmanned drone.

\section{RESULTS AND DISCUSSION}

\section{Flow Simulation and Analysis of Target Drone}

Based on the flow patterns of the hovering quadrotor drone that were observed using dry ice spray, as presented in Fig. 5, the dry ice vapor initially gathered above the drone and then accelerated rapidly downwards. To quantify exactly the intensity of turbulence and its distancedistribution as a result of the rotating propellers of the hovering drone, flow simulation software was used.

The velocity and intensity of the turbulence of the flow field around the hovering quadrotor drone were analyzed at the propellers' angular velocity of 5,700 rpm. Fig. 6 shows the turbulence intensity generated by the drone. The turbulence intensity in the drone tail area reached $36.7 \%$, as indicated by the light green stream lines, while that around the head of drone was less than $0.3 \%$, as indicated by the deep blue stream lines. Based on the results of the flow simulation, the area at the head of the drone was the best for installing air samplers. 


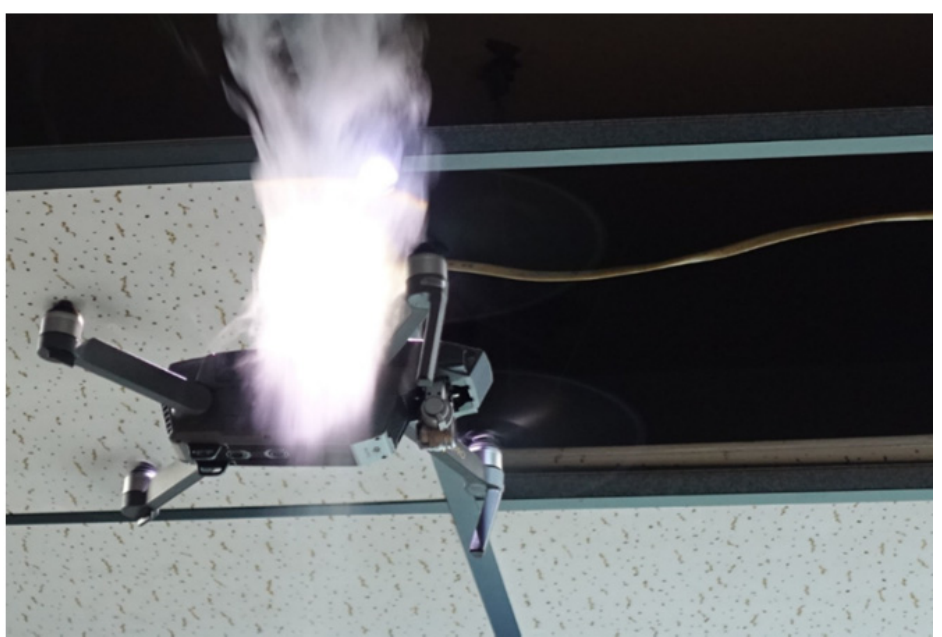

Fig. 5. Photograph of dry-ice spray streams around the hovering quadrotor drone.
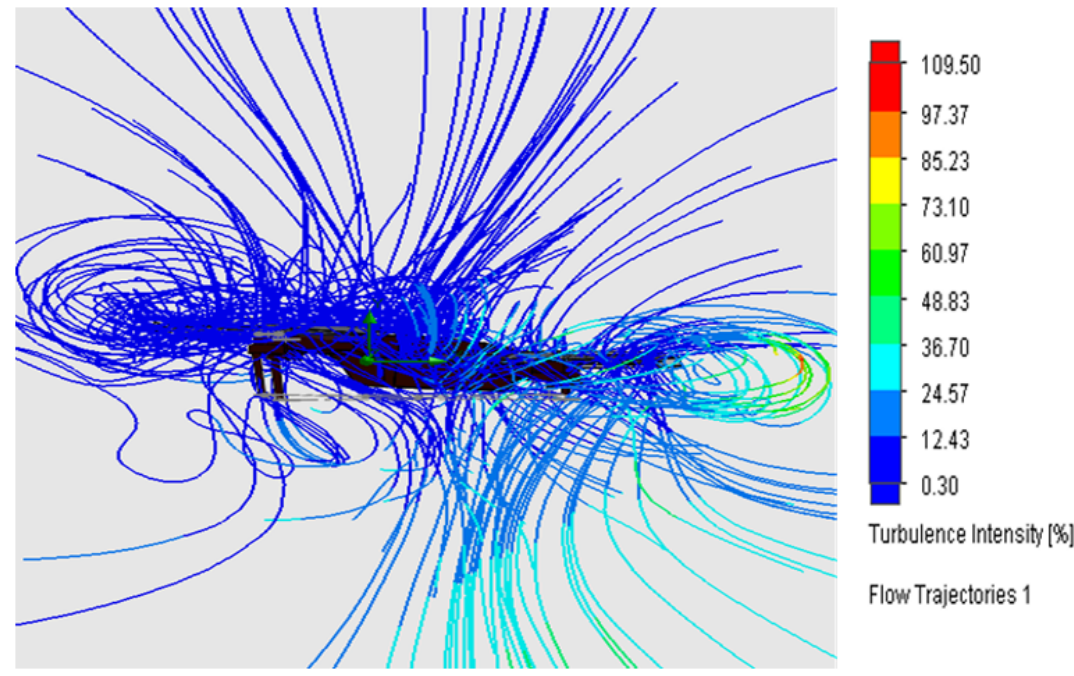

Flow Trajectories 1

Fig. 6. Diagram of the turbulence intensity of the quadrotor drone using the flow simulation software.

\section{Optimization of Sampler Location on a Hovering Drone}

Fig. 7 plots the velocity contours of the hovering drone. To confirm the reliability of the simulated velocity, the actual air velocity around the drone was measured. According to Fig. 7, the velocity at $300 \mathrm{~mm}$ (6 times the 50 -mm scale bar in Fig. 7) from the bottom center of the drone was $1.778 \mathrm{~m} \mathrm{~s}^{-1}$, which falls within the range of the measured values of $1.6-2.0 \mathrm{~m} \mathrm{~s}^{-1}$.

Fig. 8 plots the distributions of velocity and intensity of turbulence for various vertical distances of the center $\left(V_{1}\right)$ and the edge $\left(\mathrm{V}_{2}\right)$ of a propeller of the target drone from the bottom of it (see Fig. 7 for the exact locations). Typically, the area $\mathrm{G}$, in which the vertical distance from 50 to $100 \mathrm{~mm}$ in Fig. 8, below the four dashed lines is optimal for sampling air, as the velocity and turbulent intensity therein are as low as $0.5 \mathrm{~m} \mathrm{~s}^{-1}$ and $2 \%$. Fig. 9 plots the variations of velocity and turbulence intensity with horizontal distance from the center of the propeller (see Fig. 7 for exact location within the drone). As shown in Fig. 9, both velocity and turbulence intensity gradually decreased as the horizontal distance increased. For keeping the balance of the drone when it is hovering, the sampling location was determined at a vertical distance of $75(50-100) \mathrm{mm}$ and a horizontal distance of $173 \mathrm{~mm}$ from the center of the propeller. Fig. 9 shows that the velocity is $0.98 \mathrm{~m} \mathrm{~s}^{-1}$ and turbulence intensity is $1.75 \%$. Fig. 3 displays the telescoping NTS sampling device on a drone that was used for collecting gas samples exhausted from a chimney.

\section{Pilot test of VOC Sampling and Analysis}

A VOC exhaust system (Fig. 3) was constructed to verify experimentally the effectiveness of the optimized telescoping NTS sampling device on the drone. The telescoping sampling device was designed to be installed in the area of low turbulence in front of the bottom of the head of the drone. Other samplings via NTS were performed beneath the drone around $5 \mathrm{~cm}$ from the center of its underside to compare the samples that were collected from the telescoping sampling device. Fig. 10 presents the performance of the NTS at the clearly optimal location on the drone, as the downward air flow drawn from rotating propellers resulted in an unsuccessful extraction of VOCs and only very small 

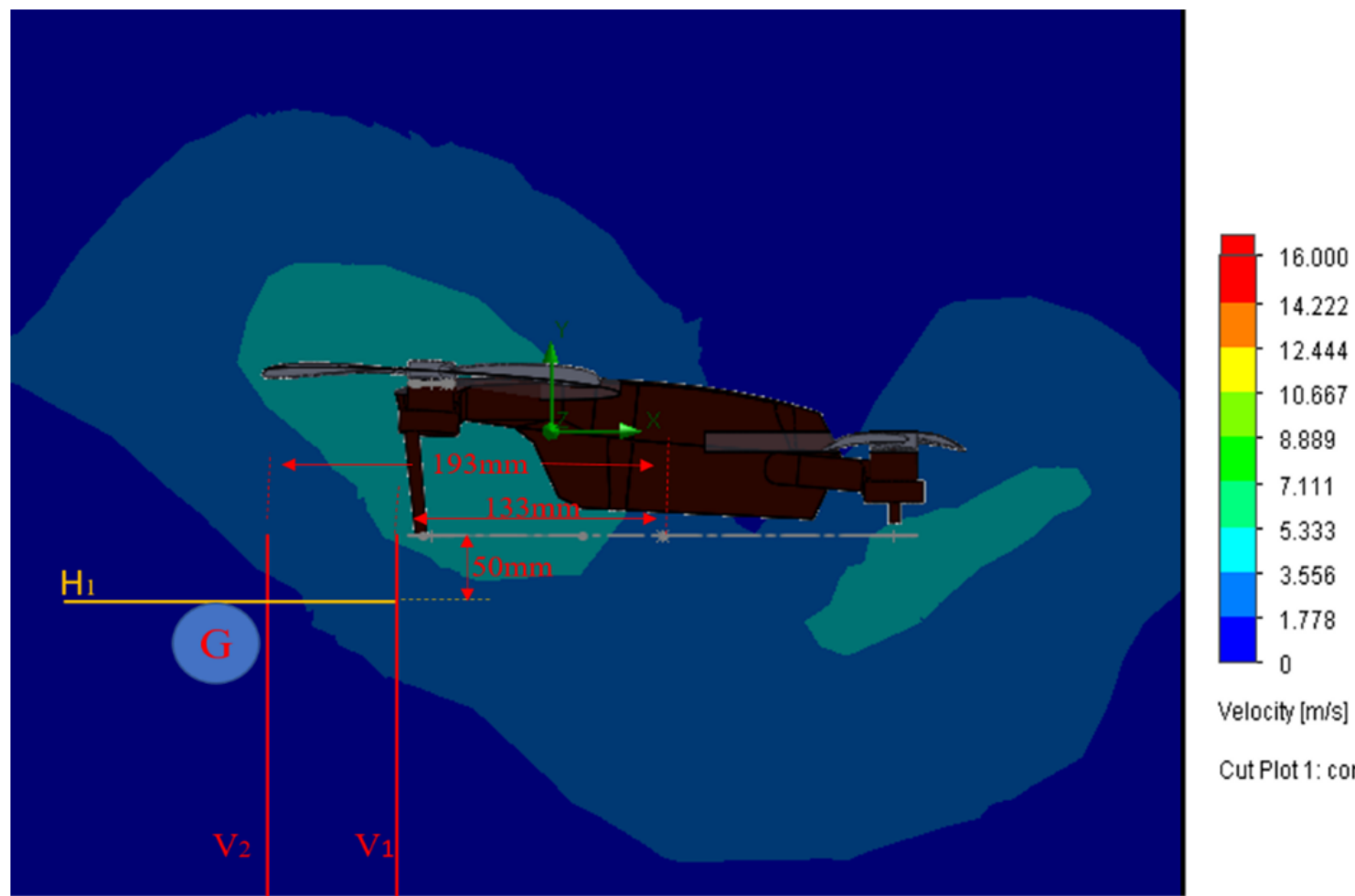

Velocity $[\mathrm{m} / \mathrm{s}]$

Cut Plot 1: contours

Fig. 7. Velocity contours of the boundary flow field around the quadrotor drone.

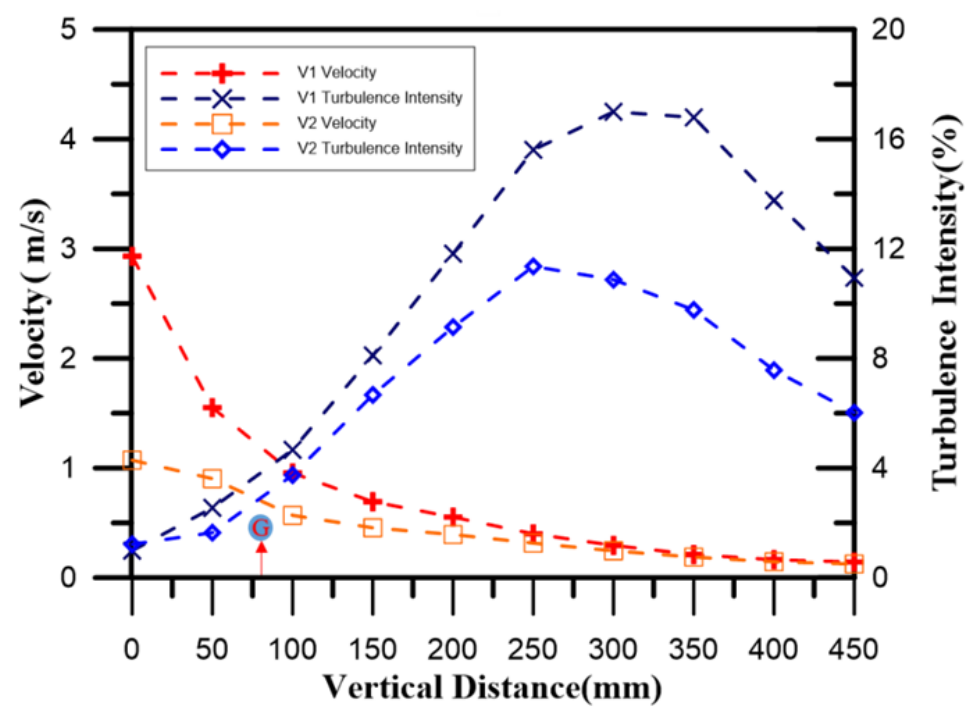

Fig. 8. Variations of velocity and turbulence intensity for the vertical boundary flow field with the vertical distance from the bottom of the drone.

peaks were analyzed by GC-FID when the NTS was equipped on the bottom of the drone. The downwardly flowing streams of air from the rotating propellers must be carefully considered to prevent the disturbance of airborne compounds.

Table 1 shows the VOC exhaust concentrations from the chimney that were obtained using different sampling/monitoring methods. The concentrations, sampled with an NTS and Teflon air bags and examined with a GC-FID, were very close to each other, so an NTS on a quadrotor drone can provide representative air samples like those collected using Teflon air bags. In practice, a drone with an NTS can sample air pollution at a high altitude, such as emissions from the pressure relief valves (PSVs) of a big reserve tank in a petrochemical factory.

\section{CONCLUSIONS}

This investigation shows that an NTS on a quadrotor drone can be used as a mobile device for sampling 


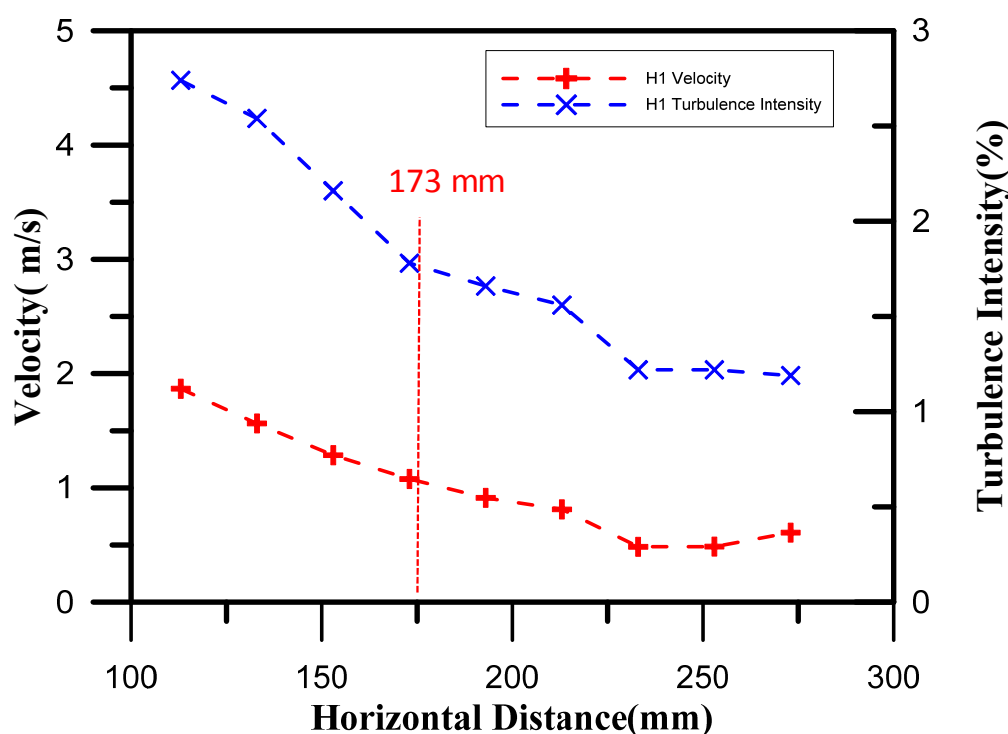

Fig. 9. Variations of velocity and turbulence intensity for the horizontal boundary flow field with the distance from the front propellers' center of the drone.

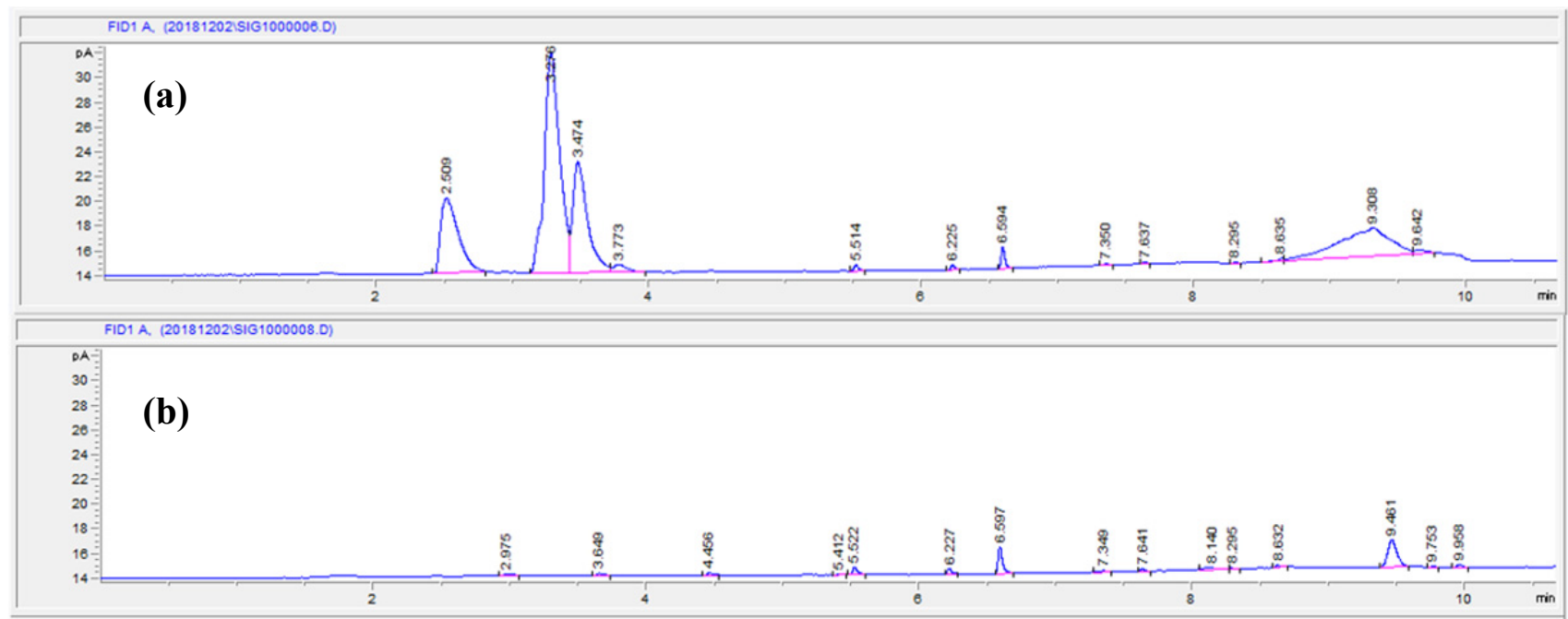

Fig. 10. (a) Chromatographic analysis diagram of VOCs extracted via the telescoping NTS on the drone. The main VOCs (and their residence times) are toluene $(2.7 \mathrm{~min})$, ethylbenzene $(3.2 \mathrm{~min})$, and $p$-xylene $(3.4 \mathrm{~min})$. (b) Very small peaks were analyzed when the NTS was equipped on the bottom of the drone.

Table 1. Comparison of VOC concentrations by using different sampling/monitoring methods.

\begin{tabular}{|c|c|c|c|c|c|}
\hline \multicolumn{5}{|c|}{ VOC concentration ranges (ppm) } & \multirow{2}{*}{$\begin{array}{l}\text { Ambient weather } \\
\text { conditions }\end{array}$} \\
\hline Collected via & on a drone & Sampling by $\mathrm{T}$ & on bags & Detecting by a monitor & \\
\hline Toluene & $142 \pm 20^{(\mathrm{a})}$ & Toluene & $140 \pm 15^{(\mathrm{b})}$ & TVOCs $1,287 \pm 202^{(\mathrm{c})}$ & See Notes ${ }^{(\mathrm{d})}$ \\
\hline Ethylbenzene & $359 \pm 23^{(a)}$ & Ethylbenzene & $327 \pm 20^{(b)}$ & & \\
\hline p-Xylene & $176 \pm 19^{(a)}$ & p-Xylene & $160 \pm 8.5^{(\mathrm{b})}$ & & \\
\hline
\end{tabular}

Notes:

(a) Mean values are for 8 NTS samplings; and the extraction duration time is 15 min per NTS sample.

(b) Mean values are for 8 Teflon bag samplings; and the collecting time is 1 min per bag sample.

(c) Continuously monitoring period is $120 \mathrm{~min}$; the reading values are the equivalent concentrations when the monitor is calibrated by isobutylene, and if the values are multiplied to the mixed correction factor 0.487 , provided by RAE Systems Inc. (2016), the corrected TEX combined concentrations are $627 \pm 98.4 \mathrm{ppm}$.

Wind speed and temperature monitoring period is $120 \mathrm{~min}$. Wind speed and temperature ranked $0.58 \pm 0.34 \mathrm{~m} \mathrm{~s}^{-1}$ and 27.3 $\pm 0.4^{\circ} \mathrm{C}$, respectively. 
atmospheric pollution at a high altitude. However, the fast downward air flow resulting from the rotation of the drone's propellers must be prevented from disturbing the distribution of ambient contaminants. We modeled the flow streams to identify the optimal position for an NTS on a drone and experimentally proved the feasibility of operating a telescoping sampling shaft.

The NTS, being a mini-sampler, is the critical apparatus in this mobile system and can be attached to the target drone along with a ventilation pump and a small circuit board for operating the pump. These accessories only total approximately $200 \mathrm{~g}$ in mass; thus, the proposed NTS system outperforms Teflon sampling bags or stainless steel canisters for drone sampling.

\section{ACKNOWLEDGEMENTS}

The authors would like to thank the Ministry of Science and Technology, Taiwan, for financially supporting this research under contract No. MOST-107-2622-E242-002CC3. Mr. Yen-Ming Tsao is appreciated for his kind assistance in assembling the sampling device on the drone.

\section{SUPPLEMENTARY MATERIAL}

Supplementary data associated with this article can be found in the online version at http://www.aaqr.org.

\section{REFERENCES}

Aurell, J., Mitchell, W., Chirayath, V., Jonsson, J., Tabor, D. and Gullett, B. (2017). Field determination of multipollutant, open area combustion source emission factors with a hexacopter unmanned aerial vehicle. Atmos. Environ. 166: 433-440.

Chang, C.C., Wang, J.L., Chang, C.Y., Liang, M.C. and Lin, M.R. (2016). Development of a multicopter-carried whole air sampling apparatus and its applications in environmental studies. Chemosphere 144: 484-492.

Chang, C.C., Chang, C.Y., Wang, J.L., Lin, M.R, Ou-Yang, C.F., Pan, H.H. and Chen, Y.C. (2018). A study of atmospheric mixing of trace gases by aerial sampling with a multi-rotor drone. Atmos. Environ. 184: 254-261.

Cheng, M.D. (2018). Selective collection of airborne particulate matter. Aerosol Air Qual. Res. 18: 13611365.

Cheng, W.H., Zhan, W. and Pawliszyn, J. (2011). Extraction of gaseous VOCs using passive needle trap samplers. Aerosol Air Qual. Res. 11: 387-392.

Cheng, W.H. and Lai, C.H. (2014). Sampling gaseous compounds from essential oils evaporation by solid phase microextraction devices. Atmos. Environ. 99: 124-129.

Cheng, W.H., Zhan, W. and Pawliszyn, J. (2013). Gaseous and particle-bound VOC products of combustion extracted by needle trap samplers. J. Chin. Chem. Soc. 60: 1027-1032.

Cheng, W.H., Jiang, J.R., Lin. C., Liou, J.J., Wu, Z.H., Hsu, Y.H. and Yang, Z.Y. (2014). Preparation of needle trap samplers to extract air compounds from indoor electric-vaporizing sources. J. Air Waste Manage. Assoc. 64: 488-493.

Cheng, W.H., Lai, C.H., Tzeng, W.J., Her, C. and Hsu, Y.H. (2015). Gaseous products of incense coil combustion extracted by passive solid phase microextraction samplers. Atmosphere 6: 822-833.

Cheng, W.H., Tsai, D.Y., Lu, J.Y. and Lee, J.W. (2016). Extracting emissions from air fresheners using solid phase microextraction devices. Aerosol Air Qual. Res. 16: 2362-2367.

Cheng, W.H., Huang, H.L, Chen, K.S. and Chang, Y.C. (2017). Quantification of VOC emissions from paint spraying on a construction site using solid phase microextraction devices. J. Environ. Sci. Health A 52: 1158-1163.

Cheng, W.H. and Wu, H.M. (2019). Assessing organic chemical emissions and workers' risk of exposure in a medical examination center using solid phase microextraction devices. Aerosol Air Qual. Res. 19: $865-870$.

Lähde, T., Niemi, J.V., Kousa, A., Rönkkö, T., Karjalainen, P., Keskinen, J., Frey, A., Hillamo, R. and Pirjola, L. (2014). Mobile particle and $\mathrm{NO}_{\mathrm{x}}$ emission characterization at helsinki downtown: comparison of different traffic flow areas. Aerosol Air Qual. Res. 14: 1372-1382.

Lord, H., Zhan, W. and Pawliszyn, J. (2010). Fundamentals and applications of needle trap devices. Anal. Chim. Acta 677: 3-18.

McGonigle, A.J.S., Aiuppa, A., Giudice, G., Tamburello, G., Hodson, A.J. and Gurrieri, S. (2008). Unmanned aerial vehicle measurements of volcanic carbon dioxide fluxes. Geophys. Res. Lett. 35: L06303.

Peng, Z.R., Wang, D., Wang, Z., Gao, Y. and Lu, S. (2015). A study of vertical distribution patterns of $\mathrm{PM}_{2.5}$ concentrations based on ambient monitoring with unmanned aerial vehicles: A case in Hangzhou, China. Atmos. Environ. 123: 357-369

RAE Systems Inc. (2016). Guideline for PID Instrument Response, Technical Note 106. Jan. 12, 2016, https://www. raesystems.com/customer-care/resource-center/tn-106-gu ideline-pid-instrument-response, Last Access: 22 Feb. 2019.

Schuyler, T.J. and Guzman, M.I. (2017). Unmanned aerial systems for monitoring trace tropospheric gases. Atmosphere 8: 206.

Solidworks (2018). Solidworks: Flow simulation technical reference 2018. SolidWorks, the United States.

Syu, J.Y., Cheng, Y.C., Kao, Y.Y., Liang, C.S., Yan, Y.L., Lai, C.Y., Chang, C.T., Chen, C.C., Young, C.Y., Wu, Y.L. and Lin, W.Y. (2016). The horizontal and vertical characteristics of Aeolian dust from riverbed. Aerosol Air Qual. Res. 16: 3026-3036.

Villa, T.F., Salimi, F., Morton, K., Morawska, L. and Gonzalez, F. (2016). Development and validation of a UAV based system for air pollution measurements. Sensors 16: 2202.

Vo, T.D.H., Lin, C., Weng, C.E., Yuan, C.S., Lee, C.W., Hung, C.H., Bui, X.T., Lo, K.C. and Lin, J.X. (2018). 
Vertical stratification of volatile organic compounds and their photochemical product formation potential in an industrial urban area. J. Environ. Manage. 217: 327336.

Xu, J., Jiang, H., Zhao, H. and Stephens, B. (2017). Mobile monitoring of personal $\mathrm{NO}_{\mathrm{x}}$ exposures during scripted daily activities in Chicago, IL. Aerosol Air Qual. Res. 17: 1999-2009.

Zhou, S., Peng, S., Wang, M., Shen, A. and Liu, Z. (2018).
The characteristics and contributing factors of air pollution in Nanjing: A case study based on an unmanned aerial vehicle experiment and multiple datasets. Atmosphere 9: 343.

Received for review, April 4, 2019

Revised, May 24, 2019

Accepted, June 6, 2019 